\title{
Reproductive Maternal and Newborn Health Providers' Assessment of Facility Preparedness and Its Determinants during the COVID-19 Pandemic in Lagos, Nigeria
}

\author{
Charles Ameh, ${ }^{1 \star}$ Aduragbemi Banke-Thomas, ${ }^{2}$ Mobolanle Balogun, ${ }^{3}$ Christian Chigozie Makwe, ${ }^{4}$ and Bosede Bukola Afolabi ${ }^{4}$ \\ ${ }^{1}$ International Public Health Department, Liverpool School of Tropical Medicine, Liverpool, United Kingdom; ${ }^{2}$ LSE Health, London School of \\ Economics and Political Science, London, United Kingdom; ${ }^{3}$ Department of Community Health and Primary Care, College of Medicine, \\ University of Lagos, Idi-Araba, Lagos, Nigeria; ${ }^{4}$ Department of Obstetrics and Gynaecology, College of Medicine of the University of Lagos, \\ Idi-Araba, Lagos, Nigeria
}

\begin{abstract}
The global COVID-19 pandemic is predicted to compromise the achievement of global reproductive, maternal, and newborn health (RMNH) targets. The objective of this study was to determine the health facility (HF) preparedness for RMNH service delivery during the outbreak from the perspective of RMNH providers and to determine what factors significantly predict this. An anonymous cross-sectional online survey of RMNH providers was conducted from to July 1-21, 2020 in Lagos State, Nigeria. We conducted a descriptive and ordinal regression analysis, with RMNH worker perception of HF preparedness for RMNH service delivery during the outbreak as the dependent variable. In all, 256 RMNH workers participated, 35.2\% reported that RMNH services were unavailable at some time since March 2020 , $87.1 \%$ felt work-related burnout, $97.7 \%$ were concerned about the availability of personal protective equipment (PPE) and related guidelines, and only $11.7 \%$ were satisfied with the preparedness of their HFs. Our final model was a statistically significant predictor of RMNH worker perception of HF preparedness explaining $54.7 \%$ of the variation observed. The most significant contribution to the model was communication by HF management (likelihood ratio chi-square [LRCS]: 87.94, $P<0.001$ ) and the availability of PPE and COVID-19 guidelines (LRCS: 15.43, $P<0.001$ ). A one-unit increase in the level of concern about the availability of PPE and COVID-19 guidelines would increase the odds of observing a higher category of satisfaction with HF COVID-19 preparedness. Adequate support of RMNH providers, particularly provision of PPE and guidelines, and appropriate communications about COVID-19 should be prioritized as part of HF preparedness.
\end{abstract}

\section{INTRODUCTION}

The WHO declared COVID-19 as a pandemic on March 11, 2020, after first being reported in Hubei Province, China, in December 2019. As of September 20, 2020, more than 30 million cases and close to 1 million deaths have been recorded globally, with Brazil, India, and the United States accounting for about $50 \%$ of the cases. ${ }^{1}$ For the same period, 1.2 million cases and more than 30, 000 deaths were reported from Africa, with the highest numbers from South Africa, Egypt, Morocco, Ethiopia, and Nigeria. ${ }^{2}$

Governments around the world have implemented various public health and social measures to reduce the spread of COVID-19. Health service provision has been modified in many settings to focus on managing COVID-19 cases, by reducing service provision for non-COVID-19 health emergencies and essential health services. Thus, health systems have struggled to cope with maintaining essential health services while trying to control the infection. ${ }^{3}$ Of all health services, reproductive, maternal, and newborn health (RMNH) services are likely to be impacted the most, like in the previous infectious disease pandemic. A systematic review of the impact of the 2014-2015 Ebola outbreak reported that the health system in affected areas collapsed because of overwhelming demand directly linked to the outbreak, health workers' death, resource diversion, and closure of facilities that comprised access to essential health services. They also reported that in Ebola-affected areas, there was an $80 \%$ reduction in maternal delivery care and increased morbidity and mortality. ${ }^{4}$

\footnotetext{
*Address correspondence to Charles Ameh, International Public Health Department, Liverpool School of Tropical Medicine, Pembroke Place, Liverpool L3 5QA, United Kingdom. E-mail: charles.ameh@ Istmed.ac.uk
}

A recent study that modeled the impact of the workforce, supplies, demand, and access reductions due to the COVID19 outbreak showed that $9.8-51.9 \%$ reduction in coverage could result in up to 113,000 additional maternal deaths (38.6\% increase from baseline) in 12 months, in 118 low- and middle-income countries. ${ }^{5}$ Sixty percent of these estimated deaths may be due to a reduction in the availability of drugs. ${ }^{5}$ Also, Riley et al. ${ }^{6}$ estimated the potential impact of the COVID19 pandemic on sexual and reproductive health in low- and middle-income countries, reporting that a $10 \%$ decline in the use of short- and long-acting contraceptives will result in 15.4 million unintended pregnancies. Although the mechanism for the reduction in supplies, demand, and access can be linked to the direct effect of various policies that restrict movement resulting in a disruption in the supply chain, household income, and travel infrastructure, the mechanism for its influence on the health workforce is not straightforward. There are several possibilities including but not limited to staff COVID-19 infection and/or death, redeployment to COVID-19 treatment units, staff discrimination of infected patients, staff refusal to treat COVID19 patients, staff experiencing community discrimination, and work-related stress resulting in absenteeism or staff off work due to a need to care for infected family members. ${ }^{3,7}$

Before the pandemic, Nigeria already contributed to about $13 \%$ of the estimated global maternal deaths annually and had an estimated maternal mortality ratio (MMR) of 556/100,000 live births. Thus, achieving the target MMR of less than 70/ 100,000 live births as part of the ending preventable maternal mortality strategy over the next 10 years appears unrealistic. ${ }^{8}$ Lagos State, in the southwestern part of Nigeria, has consistently recorded the highest number of confirmed COVID-19 cases and deaths since the pandemic. ${ }^{9}$ Now within the context of the COVID-19 pandemic, the sufficiency of RMNH services in a state like Lagos, which is the epicenter of the disease, comes into sharp focus. 
With the health workers being the ones at the front line of fighting COVID-19, their perception of facility preparedness is particularly critical. Semaan et al. ${ }^{10}$ conducted a global crosssectional study earlier on in the pandemic (between 24 March and April 10, 2020), and documented the experiences of frontline RMNH workers in 81 countries and had 714 respondents $(47 \%$ from LMICs including $16 \%$ from subSaharan Africa [SSA]). The key results were that LMIC-based respondents were worried about lack of access to evidence on COVID-19 in pregnancy, low perceived knowledge to care for COVID-19 maternity patients, a low proportion of institutions providing relevant COVID-19 training, and low availability of MNH COVID-19 guidelines. In addition, almost four in 10 respondents reported substantially higher stress levels, and there was a significant concern about $\mathrm{MNH}$ staff safety. ${ }^{10}$ Although this study had a small sample size per country and did not explore the determinants of facility preparedness for RMNH services, it provided a snapshot of the preparedness, the response of various health systems, and the effect of the COVID-19 pandemic in the initial phase of this evolving outbreak from healthcare providers' perspective.

Early on during the pandemic, the WHO produced guidelines with regular updates for managing COVID-19 and for maintaining essential health services, and these have been adopted by many countries in SSA or similar ones produced to strengthen the responsiveness of their health system. ${ }^{3,11,12}$ The guidelines contain key recommendations to optimize health force capacity, including recruitment, repurposing within the limits of training and skills, redistributing roles among health workers while keeping health workers safe, and providing mental and psychosocial support. It is unclear to what extent these guidelines have been implemented in health facilities and how this has affected health provider perception of their facility readiness to manage COVID-19. Other studies assessing preparedness to manage COVID-19 assessed health workers knowledge, attitude, and practice, but did not explore the complex interaction among fear, anxiety, stress, support systems, and health facility (HF) preparedness. ${ }^{13-15}$

The objective of this study was to assess the preparedness of the health system in Lagos State, Nigeria, for the COVID-19 outbreak based on the perception of RMNH providers, and to determine what factors (work-related stress, training, support and coping strategies/support mechanisms, availability of personal protective equipment (PPE) and guidelines, and attendance for $\mathrm{RMNH}$ services) significantly predict this. It is expected that this study will generate context-specific data to improve the responsiveness of the health system for RMNH services and to minimize predicted adverse impact of the pandemic.

\section{METHODS}

An anonymous online cross-sectional survey of health workers providing RMNH services in Lagos State, Nigeria, was conducted from to July 1-21, 2020.

Study site and population. Lagos State is the commercial nerve center of Nigeria, located in the southwestern region with an estimated population of more than 20 million mostly urban residents and an annual growth rate of $3.2 \% .^{16}$ The total fertility rate in the state is between 3.4 and 4.2 (national average 5.3 ), $86.4 \%$ of women use a skilled health provider for antenatal care (national average 67\%), and between $61 \%$ and $77 \%$ of women have HF birth (national average $39 \%){ }^{8}$
Health is provided via health facilities owned by the federal government (teaching hospitals and federal medical centers), state government (state specialist, and teaching and general hospitals), local government (primary healthcare facilities), and private/faith-based healthcare facilities. There are 306 primary healthcare centers, 27 registered general hospitals, 4,421 private hospitals/specialist clinics/laboratories/diagnostic centers, and five tertiary health facilities. Most of these facilities provide RMNH services. ${ }^{17}$

The study population was RMNH workers in Lagos state health facilities. Health workers who do not provide RMNH care were excluded.

Sample size. Using the recommendation by Hosmer et al. ${ }^{18}$ of a minimum sample for ordinal regression analysis of 10 observations per independent variable was needed. Twentyfour independent variables were included in the questionnaire, so a minimum of 240 respondents was required. ${ }^{18}$

Data collection tool. A pretested self-administered online questionnaire in English language with six sections and 33 mandatory questions was used. Thirty RMNH workers at all levels of care (public and private) completed the questionnaire and provided additional information via telephone interviews during the pilot study. After the pilot study, the questionnaire was refined, and the average time for completion was determined. Data from the pilot study were excluded from the final analysis.

Section 1 of the survey tool used contained a question to confirm the provision of care in RMNH since March 2020, study information, and consent questions. Non-RMNH workers and RMNH workers who did not consent to the study could not proceed to Section 2 . Section 2 collected demographic data such as gender, age-group, professional cadre, type of facility, and information on a management role if any. Section 3 collected information on the impact of COVID19 on the availability and provision of RMNH services since March 2020, and reasons for these. Two of the sections had subscales for work-related stress and work-related burnout. Section 4 had six COVID-19 work-related stress questions, with four questions on a subscale with the following options: 1 ) not at all/to a very low degree, 2) seldom or to a low degree, 3) sometimes or somewhat, 4) often or to a high degree, and 5) always/to a very high degree. Section 5 had seven COVID 19 work-related burnout questions, on a subscale with the following options: one (never/almost never/to very low degree) to five (always/to a very high degree). The burnout questions were from the Copenhagen Burnout Inventory (CBI), a validated questionnaire with three subdimensions: personal burnout, work-related burnout, and client-related burnout. The three separate parts of the CBI questionnaire were designed to be applied in different domains. ${ }^{19}$ Section 6 had eight questions on support and stress-coping systems/ mechanisms and overall perception of HF readiness with options from zero (unprepared to manage COVID-19 cases) to five (extremely well prepared to manage COVID-19 cases). In all, the data collection tool used in this study had 24 independent variables (17 categorical and seven ordinal).

As part of the questionnaire development process, we analyzed the reliability and internal consistency of both scales (work-related stress and work-related burnout) in the questionnaire using the Cronbach alpha coefficient and the interitem correlation. Both scales have less than 10 questions, so a Cronbach alpha coefficient of 0.5 and inter-item correlation of 
0.2-0.4 was acceptable. ${ }^{20}$ Any item in each subscale that adversely affected the Cronbach alpha coefficient was not included in the ordinal regression analysis. ${ }^{20}$

Both subscales in the questionnaire were found to be reliable and internally consistent. The stress scale had four items, Cronbach's alpha was 0.743 , the inter-item correlation 0.425 , and the corrected item-total correlation for each item was greater than 0.4. The work burnout scale had seven items; we reverse-coded one negative item on the scale (energy for family and friends), Cronbach's alpha was 0.869 , the interitem correlation 0.470 , and the corrected item-total correlation for each item was greater than 0.038 .

Data collection. A link to the survey was shared with health workers working in Lagos State via multiple social media platforms including Facebook and WhatsApp groups of health facilities and professional associations. Reminders were sent on these platforms every 3 days to maximize the reach of the survey.

Data analysis. Four of the included independent variables were re-coded so that the reference category or ordinal scale was appropriate: availability of training on stress-coping mechanism (unsure $=0$, no $=1$, and yes $=2$ ), concern about the availability of PPE and COVID-19 guidelines (extremely concerned $=1$ and unconcerned $=5$ ), worry about community discrimination/stigma (extremely worried $=1$ and not worried $=5$ ), and $\mathrm{RMNH}$ services unavailable at any time since March 2020 (unsure $=0$, no $=1$, and yes $=2$ ).

We performed a descriptive analysis of independent variables: gender, age, professional cadre, facility type, and management role, and summarized outputs by frequency table, and cross-tabulation (facility type by the availability of RMNH services since March 2020, work colleague tested positive for COVID-19, off work due to suspected or confirmed COVID-19, work colleague died from COVID-19 disease, had COVID-19 training, availability of COVID-19 guidelines, availability of protocols for staff exposed to COVID-19 case, and awareness of COVID-19 priority testing in a HF). We used charts to describe the reasons for unavailability of, and reduced attendance for $\mathrm{RMNH}$ services between March and July 2020, as well as the frequency of coping/support mechanisms at health facilities. We also described the concern of healthcare workers about being infected at work, infecting family and friends, community stigmatization/discrimination, availability of PPE, and feeling of burnout.

We performed an ordinal regression analysis with healthcare worker perception of COVID-19 HF preparedness (1 = extremely unsatisfied with HF preparedness, 2 = very unsatisfied, 3 = neither satisfied nor unsatisfied, 4 = very satisfied, and $5=$ extremely satisfied) as the outcome or dependent variable. We used the SPSS Polytomous Logit Universal Model (PLUM) logistic regression and general linear model (GLM) programs for the ordinal regression analysis (IBM Corp. Released 2017. IBM SPSS Statistics for Windows, version 25.0, IBM Corp, Armonk, NY).

The assumptions of ordinal regression, which are the absence of multicollinearity and proportional odds, were assessed. We assessed multicollinearity by calculating variance inflation factors (VIFs). Variance inflation factor values greater than five are of concern, and 10 will suggest the presence of multicollinearity. ${ }^{21}$ We only included variables with VIFs less than five in the final ordinal regression analysis. The proportional odds assumptions, also known as the assumption of parallel lines, assess if the slope of the log-odds is equal for all categories of the dependent variable. If proportional odds cannot be assumed, then each predictor will have as many coefficients as thresholds in the ordinal regression. If the assumption of parallel lines is met, then only one coefficient needs to be calculated for each predictor. ${ }^{22}$

We used the "model fitting information" analysis to determine if the model improves our ability to predict the outcome/independent variable by comparing the -2 loglikelihood of the final model with the intercept-only model. A statistically significant $(P<0.05)$ chi-square statistic indicates that the final model gives a significant improvement over the baseline intercept-only model. The overall model significance for the ordinal logistic regression was examined using the $x^{2}$ omnibus test of model coefficients (GLM analysis). McFadden's $R^{2}$ and Nagelkerke value were examined to assess the per cent of variance accounted for by the independent variables (PLUM analysis). McFadden's $R^{2}$ values greater than 0.2 are indicative of models with excellent fit. ${ }^{23}$ Predicted probabilities of an event occurring were determined by $\operatorname{Exp}(\mathrm{B})$, also known as the odds ratio (GLM analysis).

Ethical considerations and approval. We provided study information and frequently asked questions to all participants. Participants were required to consent to the study before completing the survey, they were free to withdraw their consent at any time, and no incentives were given. Respondents who were ineligible or were eligible but did not give consent could not proceed to the survey. Ethics approval for the study was obtained from the Health Research and Ethics committee of College of Medicine University of Lagos (NHREC/19/08/ 2019B) and the Research and Ethics Committee of the Liverpool School of Tropical Medicine (20/052).

\section{RESULTS}

Three hundred sixty-three health workers in Lagos State responded to the invitation to participate in the study, but $70.5 \%$ (256) were eligible to participate because they provided $\mathrm{RMNH}$ services since March 2020. All eligible participants consented to and completed the survey.

Descriptive statistics. Most respondents were female (72\% or 184 ), aged $41-50$ years (38\% or 96 ), medical officers/ registrars/house officers (40\% or 101), from state Ministry of Health secondary facilities (52\% or 133), and $63.7 \%$ (163) had a management role (Table 1$)$.

Availability of reproductive, maternal, and newborn health services between March and July 2020. Only $35.2 \%$ (90) reported that RMNH services were unavailable at any time between March and July 2020 in their HF. Respondents reported that the two most common predominant contributions to reduced availability of RMNH services were community fear of infection from health facilities $(37.8 \%$ or 34$)$ and movement restrictions (30\% or 27 ). Less than $10 \%$ (seven) of respondents reported reduced availability of drugs and supplies and diversion of resources to treat COVID-19 patients as predominant reasons for the unavailability of RMNH services between March and July 2020 (Figure 1).

Perceived effect of the COVID-19 outbreak on attendance for RMNH services if they were available since March 2020. Of the 125 respondents who reported that $\mathrm{RMNH}$ services were available, $97.6 \%$ (122) reported a 
TABLE 1

Demographic characteristics of respondents $(n=256)$

\begin{tabular}{|c|c|c|}
\hline Variable & Categories & Frequency, $N(\%)$ \\
\hline \multirow[t]{3}{*}{ Gender } & Female & $184(72.4)$ \\
\hline & Male & 70 (27.3) \\
\hline & Not stated & $2(0.8)$ \\
\hline \multirow[t]{5}{*}{ Age-group (years) } & $21-30$ & $35(13.7)$ \\
\hline & $31-40$ & $86(33.6)$ \\
\hline & $41-50$ & $96(37.5)$ \\
\hline & $51-60$ & $36(14.1)$ \\
\hline & Older than 60 & $3(1.2)$ \\
\hline \multirow[t]{5}{*}{ Professional cadre } & Community health officer/CRH worker & $23(9)$ \\
\hline & Consultant & $41(16)$ \\
\hline & Nurse/midwife & $89(34.8)$ \\
\hline & Medical officer/registrar/house officer & $101(39.5)$ \\
\hline & Others & $2(0.8)$ \\
\hline \multirow[t]{5}{*}{ Facility type } & Primary care (dispensary/PHC) & 39 (15.2) \\
\hline & State govt. secondary care (state hospital) & $133(52)$ \\
\hline & Fed. govt. tertiary care (FMC/teaching hospital) & $60(23.4)$ \\
\hline & Private/mission hospital & $23(9)$ \\
\hline & Others & $1(0.3)$ \\
\hline \multirow[t]{2}{*}{ Management role } & Yes & $163(63.7)$ \\
\hline & No & $93(36.3)$ \\
\hline \multirow[t]{6}{*}{ Management role type } & $\mathrm{HoD} / \mathrm{dHoD}$ & $45(17.6)$ \\
\hline & $\begin{array}{l}\text { Medical director/chief medical officer/medical officer of health/chief } \\
\text { medical officer }\end{array}$ & $28(10.9)$ \\
\hline & LG/state $\mathrm{MoH}$ rep. or health manager & $3(1.2)$ \\
\hline & $\begin{array}{l}\mathrm{CNO} / \text { Apex nurse. } \mathrm{CHO} / \text { ward matron/hospital matron/coordinator/ward } \\
\text { manager }\end{array}$ & $69(27)$ \\
\hline & Others & $18(7)$ \\
\hline & Not applicable & $93(36.3)$ \\
\hline
\end{tabular}

reduction in attendance for these services, 51.2\% reported less than $50 \%$ reduction, and $31.2 \%$ (39) reported more than $50 \%$ reduction in attendance.

The perceived contributions to reduced attendance for $\mathrm{RMNH}$ care when these services were available were similar to the reasons for nonavailability of these services reported earlier. The two most common predominant contributions to reduced availability of RMNH services were community fear of infection from health facilities $(47.6 \%)$ and movement restrictions (47.6\%). Reduced availability of drugs and supplies and diversion of resources to treat COVID-19 patients were not considered by the HCPs to have affected the availability of RMNH services (Figure 2).

Concern about being infected at work, infecting family and friends, community stigmatization/discrimination, availability of PPE, and feeling of burnout. Ninety-five per cent (244) of all respondents were worried about being infected with coronavirus from work, and 94.9\% (243) were concerned about infecting friends and family because of their exposure to COVID-19 at work. Eighty-two per cent (210) of respondents were worried about community stigmatization or discrimination as a result of their potential
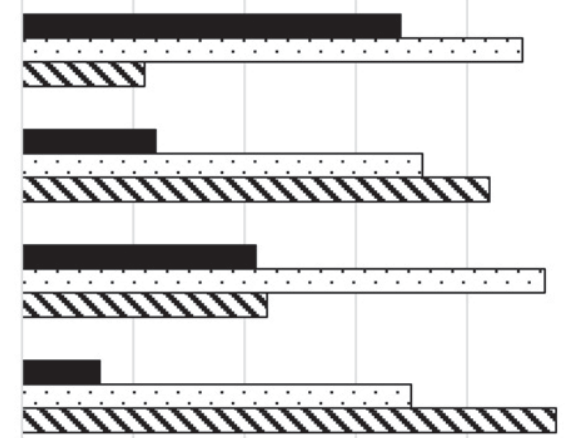

Movement restrictions

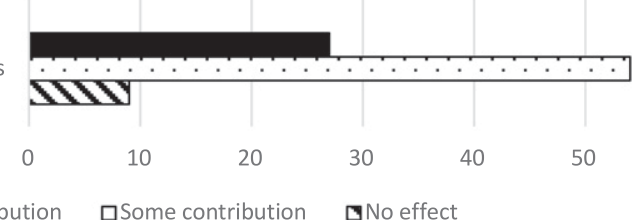

FIGURE 1. Reasons for unavailability of reproductive, maternal, and newborn health services since March 2020. 


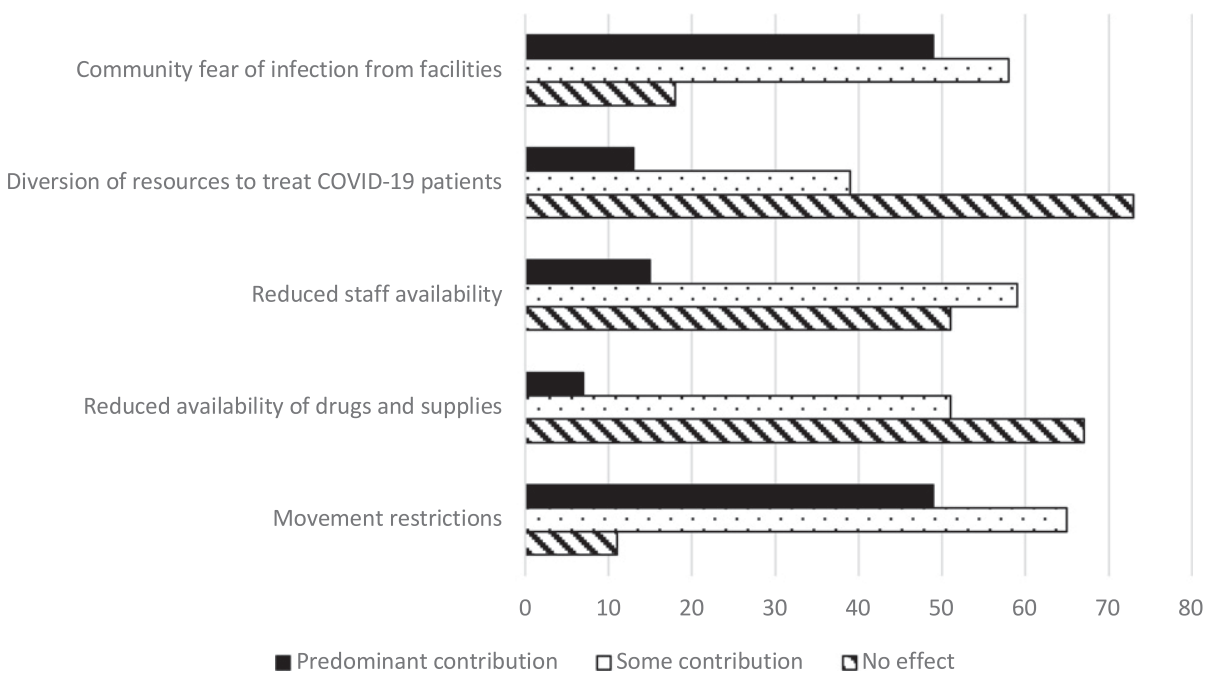

FIGURE 2. Reasons for reduced attendance for reproductive, maternal, and newborn health services.

exposure to COVID-19 cases in their facilities. Most respondents $(97.7 \%)$ were concerned about the availability of PPE and related guidelines at work, and $87 \%$ (223) experienced work-related burnout since the COVID-19 outbreak (Table 2).
Support systems to cope with providing care during the pandemic. Most of the respondents $(73.8 \%$ or 189$)$ had attended training on how to respond to COVID-19, and $82.4 \%$ (211) had COVID-19 guidelines in their facilities. About $67 \%$ (171) reported that protocols for staff exposed to COVID-19

TABLE 2

Cross-tabulation of selected variables

\begin{tabular}{|c|c|c|c|c|c|c|c|}
\hline & & & & Facility & pe, $N(\%)$ & & \\
\hline Variable & & PHC & $\begin{array}{l}\text { State MoH secondary } \\
\text { care hospital }\end{array}$ & $\begin{array}{l}\mathrm{FMOH} / \mathrm{SMoH} \\
\text { tertiary } \\
\text { care hospital }\end{array}$ & $\begin{array}{l}\text { Private/faith-based/ } \\
\text { NGO hospital }\end{array}$ & $\begin{array}{l}\text { Others (research } \\
\text { institute) }\end{array}$ & Total \\
\hline $\mathrm{RH}$ services unavailable since March & No & $26(66.7)$ & 73 (54.9) & $13(21.7)$ & $12(52.2)$ & $1(100)$ & $125(48.8)$ \\
\hline 2020 & Yes & $8(20.5)$ & $37(27.8)$ & $37(61.7$ & $8(34.8)$ & $0(0.0)$ & $90(35.2)$ \\
\hline & Unsure & $5(12.8)$ & $23(17.3)$ & $10(16.6)$ & $3(13)$ & $0(0.0)$ & $41(16)$ \\
\hline Off-work because of suspected or & No & 31 (79.5) & 96 (72.2) & $39(65)$ & $18(78.3)$ & $0(0.0)$ & $185(72.5)$ \\
\hline confirmed COVID-19 infection & Yes & $8(20.5)$ & $37(27.8)$ & $21(35)$ & $5(21.7)$ & $1(100)$ & $70(27.5)$ \\
\hline Work colleague positive for COVID-19 & No & $24(61.5)$ & 43 (32.3) & $13(21.7)$ & $14(60.9)$ & $0(0.0)$ & $94(36.7)$ \\
\hline & Yes & $15(38.5)$ & $90(67.7)$ & 47 (78.3) & $9(39.1)$ & $1(100)$ & $162(63.3)$ \\
\hline Colleague off-work because of suspected & No & $20(51.3)$ & $41(30.8)$ & $11(18.3)$ & $5(21.7)$ & $0(0.0)$ & $77(30.1)$ \\
\hline or confirmed COVID-19 infection & Yes & $19(48.7)$ & $92(69.2)$ & $49(81.7)$ & 18 (78.3) & $1(100)$ & 179 (69.9) \\
\hline Work colleague died from COVID-19 & No & 36 (92.3) & $118(88.7)$ & $54(90.4)$ & $22(95.7)$ & $1(100)$ & 231 (90.2) \\
\hline disease & Yes & $3(7.7)$ & $15(11.3)$ & $6(10)$ & $1(0.4)$ & $0(0.0)$ & $25(9.8)$ \\
\hline Worried about potentially infecting friends & No & $5(12.8)$ & $4(3)$ & $2(3.3)$ & $2(8.7)$ & $1(100.0)$ & $13(5.1)$ \\
\hline and family via work exposure* & Yes & $34(87.2)$ & $129(97)$ & $58(96.7)$ & 21 (91.3) & $0(0.0)$ & $243(94.9)$ \\
\hline Worried about being infected ${ }^{*}$ & No & $4(10.3)$ & $4(3)$ & 3 (5) & $1(4.3)$ & $0(0.0)$ & $12(4.7)$ \\
\hline & Yes & $35(89.7)$ & $129(97)$ & $58(96.7)$ & 21 (91.3) & $1(100.0)$ & 244 (95.3) \\
\hline Worried about COVID-19-related & No & $6(15.4)$ & $23(17.3)$ & $14(23.3)$ & $2(8.7)$ & $0(0.0)$ & $46(18)$ \\
\hline $\begin{array}{l}\text { community stigmatization/ } \\
\text { discrimination* }\end{array}$ & Yes & $33(84.6)$ & $110(82.7)$ & $46(76.7)$ & $21(91.3)$ & $1(100.0)$ & $210(82)$ \\
\hline Concerned about availability of personal & No & $2(5.1)$ & $0(0.0)$ & $2(3.3)$ & $0(0.0)$ & $1(100.0)$ & $6(2.3)$ \\
\hline protective equipment and guidelines* & Yes & 37 (94.9) & $133(100.0)$ & $58(96.7)$ & $23(100.0)$ & $0(0.0)$ & $250(97.7)$ \\
\hline Feeling of burnout since COVID-19 & No & $8(20.5)$ & $13(9.8)$ & $10(16.7)$ & $4(17.4)$ & $0(0.0)$ & $33(12.9)$ \\
\hline outbreak* $^{*}$ & Yes & 31 (79.5) & $120(90.2)$ & $50(83.3)$ & 19 (82.6) & $1(100.0)$ & $223(87.1)$ \\
\hline Attended COVID-19 training & No & $9(23.1)$ & $36(27.1)$ & $14(23.3)$ & $8(34.8)$ & $0(0.0)$ & $67(26.2)$ \\
\hline & Yes & 30 (76.9) & 97 (72.9) & $46(76.7)$ & $15(65.2)$ & $1(100)$ & $189(73.8)$ \\
\hline Availability of COVID-19 guidelines & No & $12(32.8)$ & $21(30.8)$ & $7(11.7)$ & $5(21.7)$ & $1(100)$ & 45 (17.6) \\
\hline & Yes & 27 (69.2) & 27 (69.2) & $53(88.3)$ & 18 (78.3) & $0(0.0)$ & $211(82.4)$ \\
\hline Availability of protocols for staff exposed & No & $16(41)$ & 46 (34.6) & $14(23.3)$ & $9(39.1)$ & $0(0.0)$ & 85 (33.2) \\
\hline to positive COVID-19 case & Yes & $23(59)$ & $87(65.4)$ & 46 (76.7) & $14(60.9)$ & $1(100)$ & $171(66.8)$ \\
\hline Awareness of COVID-19 priority testing in & No & 17 (43.6) & 74 (55.6) & $23(38.3$ & $13(56.5)$ & $1(100)$ & 127 (49.6) \\
\hline $\mathrm{HF}^{\star}$ & Yes & $22(56.4)$ & $59(44.4)$ & 37 (61.7) & $10(43.5)$ & $0(0.0)$ & $129(50.4)$ \\
\hline Health facility sufficiently prepared for & No & $34(87.2)$ & $117(88)$ & $55(91.7)$ & $19(82.6)$ & $1(100)$ & $226(88.3)$ \\
\hline COVID-19* & Yes & $5(12.8)$ & $16(12)$ & $5(8.3)$ & $4(17.4)$ & $0(0.0)$ & $30(11.7)$ \\
\hline
\end{tabular}


was available, but only $50.4 \%$ (129) were aware of any priority COVID-19 testing for health workers (Table 2).

About 54\% (137) of respondents reported empathy from HF management, $46.8 \%$ (120) had been trained on coping with stress and improving mental health since the outbreak, $46.4 \%$ (119) reported the availability of counseling services, and $43.4 \%$ (111) reported that resources to cope with stress and improve mental health such as mobile apps, documents, and websites since the outbreak had been shared at their facility (Figure 3).

Respondents also reported multiple individual coping strategies for work-related stress. These included prayer: $69.1 \%$ (177), discussion with colleagues and family members: $71.5 \%$ (183), rest and meditation: $66 \%$ (169), exercise: $30.5 \%$ (78), and engaging with support groups via social media: 75 (29.3\%).

Reproductive, maternal, and newborn health care provider perception of HF readiness. About $88 \%$ (226) of $\mathrm{RMNH}$ care providers did not feel their HF was sufficiently prepared. Eighty-eight per cent (117) and $91.7 \%$ (55) of all respondents from state secondary health facilities and federal government tertiary health facilities, respectively, reported that their facilities were not sufficiently prepared to manage COVID-19 cases (Table 2).

Ordinal logistic regression. An ordinal logistic regression analysis was conducted to determine if the odds of observing each response category of health worker perceived HF preparedness could be explained by the variation in the independent variables included (Table 1).

Compliance with ordinal regression analysis assumptions. Multicollinearity. Only 13 independent variables (three ordinal and 10 nominal) of the 24 in the questionnaire were included in the regression analysis because they have VIFs less than 5 , meaning that there is no high correlation between the independent variables included in the analysis (Table 3).

Test of parallel lines. The null hypothesis states that the location parameters or slope coefficients are the same across response categories; therefore, lines of the same slope are parallel. The -2 log-likelihood of the null hypothesis is 576.102 , for the general model $530.554, X^{2} 45.548, \mathrm{DF}=72$, $P=0.994$. Therefore, we failed to reject the null hypothesis, and the proportional odds assumption of our model holds.

Model fitting. The -2 log-likelihood for a model with intercept only (a model that does not control for any predictor or independent variable and simply fits an intercept to predict the outcome or dependent variable) was 764.685, whereas that for the final model was $576.102, X^{2}$ of $188.583, \mathrm{DF}=24, P<0.001$. Therefore, we reject the null hypothesis and conclude that the final model gives a significant improvement over the baseline intercept-only model. This was consistent with the results of the Ominus test from the GLM analysis: the full model was a significant improvement in fit over the null hypothesis (no predictors) $\left(\mathrm{X}^{2}[24]=188.583, P<0.001\right)$.

Pseudo $\boldsymbol{R}^{\mathbf{2}}$ (link function-logit). The link function-logit McFadden's $R^{2}$ and Nagelkerke value were used as measures of "goodness of fit" because the outcome variable output was normally distributed. The McFadden $R^{2}$ value calculated for this model was 0.242 . The Nagelkerke value was 0.547 , meaning that up to $54.7 \%$ of the variation in the outcome variable is explained by the model.

Parameter estimates. The test of model effects (GLM analysis) showed that only two independent variables had a statistically significant effect on the level of perception of facility preparedness, likelihood ratio chi-square ( $P$-value): concern about the availability of PPE and COVID-19 guidelines 15.430, df: $4(P=0.004)$, and the level of satisfaction with HF management communication on COVID-19 87.941, df: 4 $(P<0.001)$ (Table 4).

The regression coefficient for any level of concern about the availability of PPE and COVID-19 guidelines less than unconcerned about the availability of PPE and COVID-19 guidelines was not significant, $B=-0.232-1.072$. However,

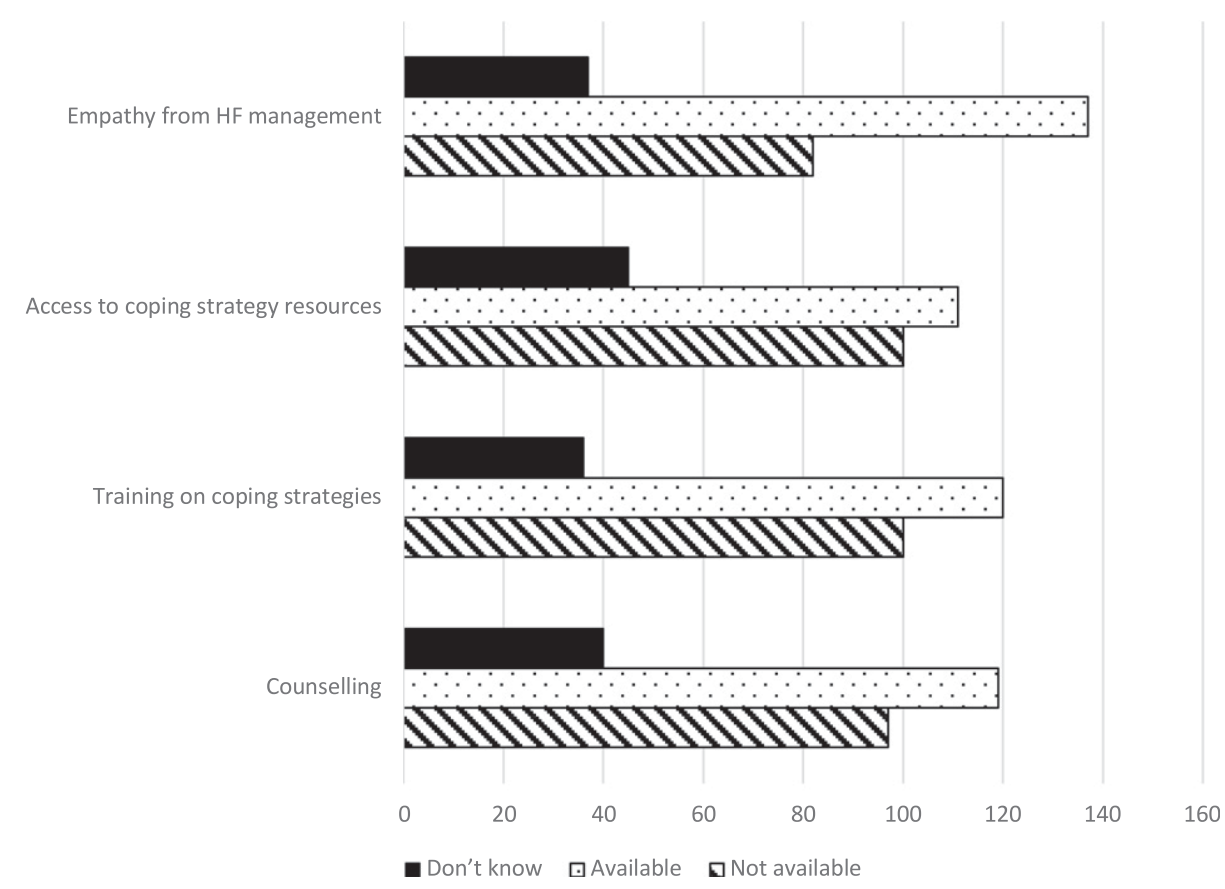

FIGURE 3. Frequency of coping/support mechanisms available at health facilities. 
TABLE 3

Variance inflation factors for independent variables included

\begin{tabular}{lc}
\hline \multicolumn{1}{c}{ Variable } & Variance inflation factor \\
\hline $\begin{array}{l}\text { Off work because of suspected or } \\
\quad \text { confirmed COVID-19 }\end{array}$ & 1.37 \\
$\begin{array}{l}\text { Work colleague tested positive for } \\
\text { COVID-19 }\end{array}$ & 2.62 \\
$\begin{array}{l}\text { Concern about the availability of personal } \\
\text { protective equipment and guidelines }\end{array}$ & 1.55 \\
$\begin{array}{l}\text { Availability of protocols for staff exposed } \\
\text { to COVID-19 case }\end{array}$ & 1.69 \\
$\begin{array}{l}\text { Level of satisfaction with HF } \\
\quad \text { communication on COVID-19 }\end{array}$ & 2.60 \\
$\begin{array}{l}\text { Availability of COVID-19 HF priority } \\
\text { testing for health workers }\end{array}$ & 1.34 \\
$\begin{array}{l}\text { Attended COVID-19 training } \\
\text { Work colleague died from COVID-19 } \\
\quad \text { disease }\end{array}$ & 1.24 \\
$\begin{array}{l}\text { Colleague off work because of COVID-19 } \\
\text { RMNH services unavailable at any time } \\
\quad \text { since March 2020 }\end{array}$ & 1.19 \\
Worries about stigma/discrimination \\
$\quad$ related to COVID-19 \\
$\begin{array}{l}\text { Availability of RMNH COVID-19 } \\
\text { guidelines }\end{array}$ \\
$\begin{array}{l}\text { Availability of training on stress-coping } \\
\text { mechanisms }\end{array}$ \\
\hline
\end{tabular}

the odds ratio of reduction in the concern about the availability of PPE and COVID-19 guidelines on the probability of a higher level of satisfaction with HF preparedness for COVID-19 was greater than 1. After controlling for all other variables, the less $\mathrm{RMNH}$ providers are concerned about the availability of PPE and guidelines, the higher the odds of a higher level of perception of HCF preparedness (Table 5).

For every one-unit increase in the level of satisfaction with the communication from HF management, there is a predicted decrease of between 1.932 and $5.806, \mathrm{x}^{2}=$ $19.339-61.155(P<0.001)$ in the log-odds of a higher level of satisfaction with the level of HF preparedness to manage COVID-19 (Table 5).

\section{DISCUSSION}

Summary of main findings. We set out to investigate the determinants of perceived HF preparedness, using ordinal regression analysis controlling for known risk factors for workrelated stress, burnout, personal preparedness (COVID-19 training), and institutional preparedness (availability of relevant guidelines, PPE, and staff support systems).

Of the $256 \mathrm{RMNH}$ service providers respondents, most worked in public-owned health facilities (91\% or 232 ), were female $(72 \%$ or 184), nurse/midwives (35\% or 89 ), and nonspecialist medical doctors (40\% or 101). About $35 \%$ (89) of respondents reported that RMNH services were unavailable at some point between March 2020 and July 2020, mostly in the tertiary public hospitals. Less than a third of RMNH workers were off work because of suspected or confirmed COVID-19 infection, and this was a lot more in secondary and tertiary public-owned hospitals than in primary health care and faith-based/private owned health facilities. $87.1 \%$ of respondents reported work-related burnout. Almost three-quarters of respondents reported that COVID-19 training was available at their health facilities; this was more available in tertiary public owned hospitals. Sixty-three percent (163), 70\% (179), and 84\% (215) of respondents were moderately or extremely concerned about being infected at work, exposing family members and friends to COVID19 , and availability of PPE and related guidelines at work. About $88 \%$ (226) of RMNH care providers did not feel their HF was sufficiently prepared.

Our final model was a statistically significant predictor of RMNH worker perception of facility preparedness after controlling for the independent variables, explaining up to $54.7 \%$ of the variation in the outcome variable. Concern about the availability of PPE and COVID-19 guidelines 15.430, df: $4(P=0.004)$, and the level of satisfaction with HF management communication on COVID-19 87.941, df: $4(P<0.001)$ were significant contributors to the model. After controlling for all other variables, the less $\mathrm{RMNH}$ providers are concerned about the availability of PPE and guidelines, the higher the odds of a higher level of perception of HCF preparedness. After controlling for all other independent variables, the more satisfied $\mathrm{RMNH}$ providers are with communications from HCF management

TABLE 4

Tests of model effects

\begin{tabular}{|c|c|c|c|}
\hline \multicolumn{4}{|c|}{ Tests of model effects } \\
\hline \multirow[b]{2}{*}{ Source } & \multicolumn{3}{|c|}{ Type III } \\
\hline & Likelihood ratio chi-square & df & Sig. \\
\hline Availability of training on stress-coping mechanisms* & 1.041 & 2 & 0.594 \\
\hline Worries about stigma/discrimination related to COVID-19* & 1.553 & 4 & 0.817 \\
\hline Off work because of suspected or confirmed COVID-19 & 0.437 & 1 & 0.509 \\
\hline Colleague off work because of COVID-19 & 1.449 & 1 & 0.229 \\
\hline Work colleague tested positive for COVID-19 & 0 & 1 & 0.986 \\
\hline Work colleague died from COVID-19 disease & 0.131 & 1 & 0.717 \\
\hline Attended COVID-19 training & 0.018 & 1 & 0.892 \\
\hline Availability of RMNH COVID-19 guidelines & 3.819 & 1 & 0.051 \\
\hline Availability of protocols for staff exposed to COVID-19 case & 0 & 1 & 0.995 \\
\hline Availability of COVID-19 HF priority testing for health workers & 3.664 & 1 & 0.056 \\
\hline Availability of personal protective equipment and COVID-19 guidelines* & 15.43 & 4 & 0.004 \\
\hline Level of satisfaction with HF communication on COVID-19 & 87.941 & 4 & 0 \\
\hline RMNH services unavailable at any time since March $2020^{*}$ & 5.542 & 2 & 0.063 \\
\hline
\end{tabular}

$\mathrm{HF}=$ health facility. Dependent variable: level of satisfaction with health facility preparedness. Model: (threshold), availability of training on stress-coping mechanisms, worries about stigma/ discrimination related to COVID-19, off work because of suspected or confirmed COVID-19, colleague off work because of COVID-19, availability of RMNH COVID-19 guidelines, availability of protocols for staff exposed to COVID-19 case, availability of COVID-19 HF priority testing for health workers, availability of COVID-19 HF priority testing for health workers, availability of COVID-19 HF priority testing for health workers, Availability of RMNH COVID-19 guidelines, level of satisfaction with HF communication on COVID-19, and level of satisfaction with HF communication on COVID-19.

${ }^{*}$ Re-coded variables. 
TABLE 5

Ordinal logistic regression results for independent variables predicting HCW perception of COVID-19 HF preparedness

\begin{tabular}{|c|c|c|c|c|c|c|c|c|c|c|}
\hline & \multirow[b]{2}{*}{ B } & \multirow[b]{2}{*}{ Std error } & \multicolumn{2}{|c|}{$95 \%$ Wald Cl } & \multicolumn{3}{|c|}{ Hypothesis testing } & \multirow[b]{2}{*}{$\operatorname{Exp}(B)$} & \multicolumn{2}{|c|}{$\begin{array}{l}95 \% \text { Wald } \mathrm{Cl} \text { for ex } \\
\text { (B) }\end{array}$} \\
\hline & & & Lower & Upper & Wald chi-square & df & Sig. & & Lower & Upper \\
\hline \multicolumn{11}{|l|}{ Threshold } \\
\hline $\begin{array}{l}\text { Satisfaction with HF preparedness = } \\
\text { extremely unsatisfied }\end{array}$ & -6.335 & 1.0214 & -8.337 & -4.333 & 38.468 & 1 & 0 & 0 & 0 & 0.013 \\
\hline $\begin{array}{l}\text { Satisfaction with HF preparedness = very } \\
\text { unsatisfied }\end{array}$ & -4.07 & 0.9897 & -6.01 & -2.131 & 16.916 & 1 & 0 & 0.02 & 0.002 & 0.119 \\
\hline $\begin{array}{l}\text { Satisfaction with HF preparedness = } \\
\text { neither satisfied or unsatisfied }\end{array}$ & -1.935 & 0.9658 & -3.828 & -0.042 & 4.013 & 1 & 0.045 & 0.14 & 0.022 & 0.959 \\
\hline $\begin{array}{l}\text { Satisfaction with HF preparedness = very } \\
\text { satisfied }\end{array}$ & 0.383 & 0.9433 & -1.466 & 2.232 & 0.165 & 1 & 0.685 & 1.47 & 0.231 & 9.32 \\
\hline \multicolumn{11}{|l|}{ Location } \\
\hline $\begin{array}{l}\text { Availability of training on stress-coping } \\
\text { mechanisms = unsure }\end{array}$ & 0.167 & 0.3997 & -0.616 & 0.951 & 0.175 & 1 & 0.675 & 1.18 & 0.54 & 2.588 \\
\hline $\begin{array}{l}\text { Availability of training on stress-coping } \\
\text { mechanisms }=\text { no* }^{*}\end{array}$ & -0.208 & 0.3187 & -0.832 & 0.417 & 0.424 & 1 & 0.515 & 0.81 & 0.435 & 1.517 \\
\hline $\begin{array}{l}\text { Availability of training on stress-coping } \\
\text { mechanisms }=\text { yes }^{\star}\end{array}$ & $0 \dagger$ & - & - & - & - & - & - & 1 & - & - \\
\hline $\begin{array}{l}\text { Worries about stigma/discrimination } \\
\text { related to COVID-19= extremely worried }\end{array}$ & 0.22 & 0.4114 & -0.587 & 1.026 & 0.285 & 1 & 0.593 & 1.25 & 0.556 & 2.79 \\
\hline $\begin{array}{l}\text { Worries about stigma/discrimination } \\
\text { related to COVID-19 = very worried }\end{array}$ & 0.568 & 0.4628 & -0.339 & 1.475 & 1.506 & 1 & 0.22 & 1.77 & 0.712 & 4.372 \\
\hline $\begin{array}{l}\text { Worries about stigma/discrimination } \\
\text { related to COVID-19= neither worried or } \\
\text { unworried }\end{array}$ & 0.276 & 0.4003 & -0.509 & 1.06 & 0.474 & 1 & 0.491 & 1.32 & 0.601 & 2.887 \\
\hline $\begin{array}{l}\text { Worries about stigma/discrimination } \\
\text { related to COVID-19= some worry }\end{array}$ & 0.26 & 0.4156 & -0.555 & 1.074 & 0.391 & 1 & 0.532 & 1.3 & 0.574 & 2.928 \\
\hline $\begin{array}{l}\text { Worries about stigma/discrimination } \\
\text { related to COVID-19= not worried }\end{array}$ & $0 \dagger$ & - & - & - & - & - & - . & 1 & - & - \\
\hline $\begin{array}{l}\text { Off work because of suspected or } \\
\text { confirmed COVID-19= no }\end{array}$ & -0.205 & 0.3099 & -0.812 & 0.403 & 0.436 & 1 & 0.509 & 0.82 & 0.444 & 1.496 \\
\hline $\begin{array}{l}\text { Off work because of suspected or } \\
\text { confirmed COVID-19= yes }\end{array}$ & $0 \dagger$ & - & - & - & - & - & - & 1 & - & - \\
\hline $\begin{array}{l}\text { Colleague off work because of COVID-19= } \\
\text { no }\end{array}$ & 0.491 & 0.4088 & -0.31 & 1.292 & 1.442 & 1 & 0.23 & 1.63 & 0.733 & 3.641 \\
\hline $\begin{array}{l}\text { Colleague off work because of COVID-19= } \\
\text { yes }\end{array}$ & $0 †$ & - & - & - & - & - & - & 1 & - & - \\
\hline $\begin{array}{l}\text { Work colleague tested positive for COVID- } \\
19=\text { no }\end{array}$ & 0.007 & 0.4093 & -0.795 & 0.81 & 0 & 1 & 0.986 & 1.01 & 0.452 & 2.247 \\
\hline $\begin{array}{l}\text { Work colleague tested positive for COVID- } \\
19=\text { yes }\end{array}$ & $0 \dagger$ & - & - & - & - & - & - & 1 & - & - \\
\hline $\begin{array}{l}\text { Work colleague died from COVID-19 } \\
\text { disease }=\text { no }\end{array}$ & 0.156 & 0.4314 & -0.689 & 1.002 & 0.131 & 1 & 0.717 & 1.17 & 0.502 & 2.723 \\
\hline $\begin{array}{l}\text { Work colleague died from COVID-19 } \\
\text { disease = yes }\end{array}$ & $0 \dagger$ & - & - & - & - & - & - & 1 & - & - \\
\hline Attended COVID-19 training = yes & 0.04 & 0.2928 & -0.534 & 0.614 & 0.018 & 1 & 0.892 & 1.04 & 0.586 & 1.847 \\
\hline Attended COVID-19 training = no & $0 \dagger$ & - & - & - & - & - & - & 1 & - & - \\
\hline $\begin{array}{l}\text { Availability of RMNH COVID-19 guidelines } \\
=\text { no }\end{array}$ & -0.746 & 0.3829 & -1.496 & 0.004 & 3.796 & 1 & 0.051 & 0.47 & 0.224 & 1.004 \\
\hline $\begin{array}{l}\text { Availability of RMNH COVID-19 guidelines } \\
=\text { yes }\end{array}$ & $0 \dagger$ & - & - & - & - & - & - & 1 & - & - \\
\hline Protocols for exposed staff available $=$ No & -0.002 & 0.3201 & -0.629 & 0.626 & 0 & 1 & 0.995 & 1 & 0.533 & 1.869 \\
\hline $\begin{array}{l}\text { Protocols for exposed staff } \\
\quad \text { available }=\text { yes }\end{array}$ & ot & - & - & - & - & - & - & 1 & - & - \\
\hline $\begin{array}{l}\text { HF priority COVID-19 testing } \\
\quad \text { available }=\text { no }\end{array}$ & -0.53 & 0.2777 & -1.074 & 0.014 & 3.642 & 1 & 0.056 & 0.59 & 0.342 & 1.014 \\
\hline $\begin{array}{l}\text { HF priority COVID-19 testing } \\
\text { available = yes }\end{array}$ & $0 †$ & - & - & - & - & - & - & 1 & - & - \\
\hline $\begin{array}{l}\text { Extremely concerned about PPE and } \\
\text { guideline availability }=1^{*}\end{array}$ & -0.232 & 0.8457 & -1.89 & 1.425 & 0.075 & 1 & 0.784 & 0.79 & 0.151 & 4.159 \\
\hline $\begin{array}{l}\text { Very concerned about PPE availability and } \\
\text { guidelines }=2^{\star}\end{array}$ & 0.92 & 0.8798 & -0.805 & 2.644 & 1.092 & 1 & 0.296 & 2.51 & 0.447 & 14.067 \\
\hline $\begin{array}{l}\text { Neither concerned nor unconcerned about } \\
\text { PPE and guideline availability }=3^{\star}\end{array}$ & 0.522 & 0.9022 & -1.246 & 2.29 & 0.335 & 1 & 0.563 & 1.69 & 0.288 & 9.879 \\
\hline $\begin{array}{l}\text { Some concern about PPE and guideline } \\
\quad \text { availability }=4.00^{*}\end{array}$ & 1.072 & 0.9672 & -0.824 & 2.968 & 1.229 & 1 & 0.268 & 2.92 & 0.439 & 19.45 \\
\hline \multirow{2}{*}{$\begin{array}{l}\text { Unconcerned about PPE and guideline } \\
\text { availability }=5.00^{\star}\end{array}$} & $0 \dagger$ & - & - & - & - & - & - & 1 & - & - \\
\hline & -5.806 & 0.7425 & -7.262 & -4.351 & 61.155 & 1 & 0 & 0 & 0.001 & 0.013 \\
\hline
\end{tabular}


TABLE 5

Continued

\begin{tabular}{|c|c|c|c|c|c|c|c|c|c|c|}
\hline & \multirow[b]{2}{*}{ B } & \multirow[b]{2}{*}{ Std error } & \multicolumn{2}{|c|}{$95 \%$ Wald $\mathrm{Cl}$} & \multicolumn{3}{|c|}{ Hypothesis testing } & \multirow[b]{2}{*}{$\operatorname{Exp}(\mathrm{B})$} & \multicolumn{2}{|c|}{$\begin{array}{l}95 \% \text { Wald } \mathrm{Cl} \text { for ex } \\
\text { (B) }\end{array}$} \\
\hline & & & Lower & Upper & Wald chi-square & df & Sig. & & Lower & Upper \\
\hline \multicolumn{11}{|l|}{$\begin{array}{l}\text { Extremely unsatisfied with } \mathrm{HF} \\
\text { management communication }=1\end{array}$} \\
\hline $\begin{array}{l}\text { Very unsatisfied with HF management } \\
\text { communication }=2\end{array}$ & -4.125 & 0.5718 & -5.245 & -3.004 & 52.038 & 1 & 0 & 0.02 & 0.005 & 0.05 \\
\hline $\begin{array}{l}\text { Neither satisfied nor unsatisfied with HF } \\
\text { management communications }=3\end{array}$ & -3.591 & 0.4831 & -4.537 & -2.644 & 55.244 & 1 & 0 & 0.03 & 0.011 & 0.071 \\
\hline $\begin{array}{l}\text { Very satisfied with HF management } \\
\text { communication }=4\end{array}$ & -1.932 & 0.4394 & -2.793 & -1.071 & 19.339 & 1 & 0 & 0.15 & 0.061 & 0.343 \\
\hline $\begin{array}{l}\text { Extremely satisfied with HF management } \\
\text { communication }=5\end{array}$ & $0 \dagger$ & - & - & - & - & - & - & 1 & - & - \\
\hline RMNH services unavailable $=$ unsure $^{\star}$ & -0.84 & 0.3878 & -1.601 & -0.08 & 4.697 & 1 & 0.03 & 0.431 & 0.202 & 0.923 \\
\hline RMNH services unavailable $=$ yes $^{*}$ & -0.043 & 0.2754 & -0.583 & 0.497 & 0.025 & 1 & 0.875 & 0.958 & 0.558 & 1.643 \\
\hline RMNH services unavailable $=$ no ${ }^{*}$ & $0 \dagger$ & - & - & - & - & - & - & & - & - \\
\hline Scale & $1 \ddagger$ & - & - & - & - & - & - & - & - & - \\
\hline \multicolumn{11}{|c|}{$\begin{array}{l}\text { HF= health facility; PPE = personal protective equipment. Dependent variable: level of satisfaction with HF preparedness. Model: (threshold), availability of training on stress-coping mechanisms, } \\
\text { worries about stigma/discrimination related to COVID-19*, off work because of suspected or confirmed COVID-19, colleague off work because of COVID-19, availability of RMNH COVID-19 } \\
\text { guidelines, availability of protocols for staff exposed to COVID- } 19 \text { case, availability of COVID-19 HF priority testing for health workers, availability of COVID-19 HF priority testing for health workers, } \\
\text { availability of COVID-19 HF priority testing for health workers, availability of RMNH COVID-19 guidelines, level of satisfaction with HF communication on COVID-19, and level of satisfaction with HF } \\
\text { communication on COVID-19*. } \\
{ }^{*} \text { Re-coded variables. } \\
\text { † Set to zero because this parameter is redundant. } \\
\text { FFixed at the displayed value. }\end{array}$} \\
\hline
\end{tabular}

on COVID-19, the lower the odds of a higher level of perception of HCF preparedness.

Interpretation. Most of our study respondents did not feel their HF was sufficiently prepared; the availability of PPE and related guidelines and satisfactory communication from HF management were significant determinants of this. This finding is interesting, given that more than $60 \%$ of them were involved with management and clinical care. Similar results were reported from a cross-sectional study conducted in Jordan (March 2020) that included only frontline medical doctors treating COVID-19 cases and doctors having institutional protocols for dealing with COVID-19 cases, and those with sustained availability of PPE reported higher scores of self-preparedness to manage COVID-19 cases. ${ }^{24}$ Following the publication of WHO guidelines to support the maintenance of essential health services in the context of COVID-19, we expected a higher perception of HF preparedness by health workers, 4 months into the pandemic.

Although our model could predict RMNH worker perception of HF preparedness after controlling for work-related stress, training, guidelines and protocols, coping strategies, PPE availability, and availability of $\mathrm{RMNH}$ services, the burnout questions could not be included in the model because of multicollinearity, but eight in 10 respondents reported workrelated burnout.

Work-related stress can affect productively if there are no active surveillance and management systems. ${ }^{7}$ More than $80 \%$ of our respondents were worried about community stigmatization due to their work; this was much less than what was reported earlier on in the pandemic from Libya. ${ }^{14}$ Elhadi et al. ${ }^{14}$ in a descriptive cross-sectional survey of obstetricians in Libya early in the pandemic reported that $20 \%$ of the respondents felt stigmatized by the community because of their work as obstetricians. The difference between this observation and ours may be due to the study population and context. Our sample had different cadre of RMNH workers from all levels of care (primary, secondary, and tertiary care). Community education, active surveillance of health workers to identify concerns, and options to manage associated stress are likely to improve the perception and experience of stigmatization, and actively manage related associated stress.

Health workers in Lagos expressed serious concerns about being infected at work or infecting family members/friends, and this is similar to findings reported during previous infectious disease outbreaks and earlier on during this ongoing outbreak. ${ }^{10,25,26}$ Such expressed fears and concerns typically lead to stress. High levels of health worker work-related stress have been reported in systematic reviews before and during the COVID-19 pandemic. ${ }^{7,27}$ The risk factors for COVID-19related physical and mental health impacts include working in a high-risk department, diagnosed family member, and improper PPE use. ${ }^{7,27}$ Most of our respondents were female; however, this is not surprising as most RMNH care providers are female. ${ }^{7}$ Shaukat et al. ${ }^{7}$ also reported that female health workers and nurses were disproportionately affected by the mental health impacts of COVID-19. ${ }^{7}$ Female health workers may have additional burden during this pandemic, caring for their families and providing care without adequate protection and support. Before the COVID-19 pandemic, Onigbogi and Banerjee $^{27}$ reported that workload, poor communication, lack of resources and equipment were significant risk factors for psychological stress in Nigerian health workers.

Preparedness can be viewed at two levels, individual preparedness and institutional preparedness. But the institution typically drives preparedness, for example, making training available to staff is likely to increase individual preparedness, providing relevant protocols, protective supplies such as PPE and support mechanisms for health workers to improve individual resilience; prevent burnout and stress; and actively support staff who suffer stress and psychological effects associated with providing care during an infectious disease outbreak. Compared with earlier on in the pandemic, training has improved, but shortages of PPE and lack of guidelines for its use are persisting problems. ${ }^{10,14,15,24}$ The improvement in training is expected following the publication of the $\mathrm{WHO}$ 
operational guidance for maintaining essential health services for the COVID-19 context and the push from the WHO, Africa CDC, Nigeria CDC, and the state and federal ministries of Health for staff training. However, this can be improved so that all health workers are regularly trained; this is critical for selfconfidence, preparedness, and resilience, and should be complemented by adequate supplies of PPE and support systems to manage work-related stress.

The improvement in training does not seem to have improved healthcare providers' perception of HF preparedness during the COVID-19 pandemic. Less than $20 \%$ of the respondents in our study reported that their facilities were sufficiently prepared to manage COVID-19 cases. This was similar to a large descriptive cross-sectional study conducted in Libya between February 26 and March 10, 2020. ${ }^{15}$ In that study, availability of PPE, awareness, and utilization of COVID-19 guidelines and protocols were low. Although this was not surprising early in the outbreak, the analysis did not explore the complex association among facility preparedness, training, work-related stress, and stress support systems.

The link between concern for PPE availability and communication from HF management regarding COVID-19 guidelines was evident from the ordinal regression analysis, and underscores the importance of caring for the carers, during a large disease outbreak. Generally, the knowledge of Nigeria health workers on COVID-19 has been reported to have improved as the pandemic progressed, ${ }^{28}$ but they need to be continually briefed and informed, covering not only up-to-date clinical management but also recognition, management, and support strategies for work-related stress and burnout. The consequences of not taking action are huge, with health worker stress and burnout, and psychological problems associated with COVID-19 can impact negatively on the provision of essential health services during a pandemic. ${ }^{10,26}$ This is a wake-up call for health system planners; our results support the urgent need to review how effective the WHO guidelines for maintaining essential health services are, and supplementary guidelines that include setting up mental health surveillance and psychosocial support for frontline health workers may be needed.

Individual-led stress-coping method/strategies reported by respondents included prayer (69.1\%), meditation (66\%), peer support (29.3\%), and exercise (30.5\%), and are not uncommon in low-resource countries. Witter et al. ${ }^{29}$ reported the use of similar coping strategies by health workers in post-conflict and infectious disease outbreak countries. Although the space to implement such individual-led coping mechanisms must be provided, including praying/meditation rooms, the role of institutional support for frontline health workers should not be minimized. Institutionalled interventions such as psychological first aid to all health workers, adequate workforce planning, regular debriefing, stress and burnout surveillance, access to psychologists and counselors, and clear and consistent communication have been recommended to minimize the risk of stress and burnout during such outbreaks. ${ }^{25,30,31}$ Although some of these interventions were reported as available by more than half of the respondents, there may be scope for improvement to proactively plan and consistently implement them in all health facilities during such outbreaks.

Our finding that RMNH services were unavailable at some point since public health and social measures were implemented in Lagos State is consistent with a nationally representative telephone survey conducted in April 2020, by the Partnership for
Evidence-Based Response to COVID-19 (PERC), which found that a high proportion of respondents who need health care have had difficulties accessing such services (39\%). The same study found that adherence to staying at home order (implemented from March 29, 2020 in Lagos State) was completely adhered to by only $23 \%$ of the population. Although it is clear that movement restrictions will have affected utilization of essential health services, healthcare worker anxiety about the risk of infection and community stigmatization may have affected the provision of services as well. Community fear of infection from health facilities was the second most common reason given by health workers for nonutilization or low attendance for RMNH services. In the subsequent PERC survey reported in September 2020, nearly three-quarters of respondents felt confident that they will get medical help if they fell ill. The change in public perception as the pandemic progressed may be associated with increasing knowledge, attitude, and practice, and lower COVID-19 risk perception by the public. ${ }^{28,32}$ Our finding of reduced attendance for RMNH services has been reported in the earlier multicountry cross-sectional study; however, with increased public knowledge and reduced risk perception, attendance for RMNH services should be expected to increase and health facilities should be prepared for this. ${ }^{10}$ It was interesting to note that in our study, diversion of resources to treat COVID-19 patients and reduced availability of medicines and supplies were perceived as having no effect or significant contribution to non-utilization or reduced attendance for RMNH services. This is contrary to the global expectations at the start of the outbreak, especially with the shutdown of international transport systems that are key for the importation of medicines and supplies. ${ }^{3,33}$ Nigeria largely depends on imports of pharmaceuticals from India and China, but there may have been sufficient stock levels of essential medicines at the initial phase of the outbreak. ${ }^{33} \mathrm{~A}$ more plausible explanation may be that as utilization of essential health services reduced, and demand for medicines and medical supplies also reduced, as such RMNH workers never appreciated the potential impact.

Strengths and limitations. There are key strengths of note in our study; this was the first to go beyond just reporting levels of preparedness to understanding the determinants of RMNH worker perception of HF preparedness and stress-coping mechanisms during the COVID-19 pandemic. Compared with the earlier studies on preparedness to manage COVID-19, we explored the interaction between determinants of preparedness that included known risk factors of health worker stress and factors that can improve preparedness using ordinal regression analysis. Our study was conducted in a COVID-19 safe way (online survey), and most respondents were in management and clinical roles and were frontline health workers, thus increasing the validity of the results.

However, our study is not without limitations. Our sample was relatively small (256) for the size of the health workforce but was more than the minimum sample size of 240 required for ordinal regression analysis based on the recommendation of Hosmer et al. ${ }^{18}$ of a minimum sample for ordinal regression analysis of 10 observations per independent variable. Our final model contained 13 variables that had no multicollinearity and low VIF (Table 5), so our sample size was sufficient. Including other variables that adequately measure work-related burnout into the model may have improved the strength of the model. Also faith-based/private-owned health facilities were underrepresented (less than 10\% all responses), but more than $85 \%$ of health facilities in Lagos are privately owned. ${ }^{17}$ Other 
studies have reported a preference for privately owned health facilities for childbirth in Lagos by about $50 \%$ of women, irrespective of social class. ${ }^{34,35}$ Therefore, our study may not be representative of the preparedness of private health facilities from the perspective of health workers, and a similar study with a large sample of private and faith-based facilities is needed to understand this.

\section{CONCLUSION}

Training of RMNH care providers, provision of PPE, guidelines, provision of support, and coping support systems complemented with appropriate communication from HF management are likely to improve the capacity of HFs to provide quality care during the COVID-19 outbreak. Similar studies are needed to evaluate the perception of preparedness in private health facilities, including the perspective of women and their carers; this is important to improve overall health system preparedness during an outbreak and for quality improvement. Full implementation of the WHO operational guidance for maintaining essential health services for the COVID-19 context should be prioritized, including monitoring of recommendations for optimizing the health workforce for a satisfactory level of HF preparedness during this pandemic.

Received October 9, 2020. Accepted for publication February 18, 2021.

Published online February 26, 2021.

Acknowledgments: We are immensely grateful to Adesola Pitan (Medical Services, Lagos State Health Service Commission), Kehinde Okunade (Society of Gynaecology and Obstetrics of Nigeria, Lagos sector), Japhet Olugbogi (Committee on COVID-19 Nigerian Medical Association, Lagos), Anthonia Onyenwenyi (CHO Training School, LUTH), Blessing Israel (National Association of Nigeria Nurses and Midwives Lagos State Council), Doyin Ogunyemi (Association of Public Health Physician of Nigeria, Lagos Chapter), and Ibironke Sodeinde (Medical Women's Association of Nigeria, Lagos Chapter) for their immense support in disseminating the survey tool used in this study. The American Society of Tropical Medicine and Hygiene has waived the Open Access fee for this article due to the ongoing COVID19 pandemic.

Disclosure: The dataset used and/or analyzed during the current study is available from the corresponding author on reasonable request.

Authors' addresses: Charles Ameh, International Public Health Department, Liverpool School of Tropical Medicine, Liverpool, United Kingdom, E-mail: charles.ameh@Istmed.ac.uk. Aduragbemi Banke-Thomas, LSE Health, London School of Economics and Political Science, London, United Kingdom, E-mail: a.bankethomas@ Ise.ac.uk. Mobolanle Balogun, Department of Community Health and Primary Care, College of Medicine, University of Lagos, Idi-Araba, Lagos, Nigeria, E-mail: mbalogun@cmul.edu.ng. Christian Chigozie Makwe and Bosede Bukola Afolabi, Department of Obstetrics and Gynaecology, College of Medicine of the University of Lagos, Idi-Araba, Lagos, Nigeria, E-mails: ccmakwe@unilag.edu.ng and bbafolabi@unilag. edu.ng.

This is an open-access article distributed under the terms of the Creative Commons Attribution (CC-BY) License, which permits unrestricted use, distribution, and reproduction in any medium, provided the original author and source are credited.

\section{REFERENCES}

1. World Health Organization, 2020. WHO Coronavirus Disease (COVID-19) Dashboard | WHO Coronavirus Disease (COVID-19) Dashboard. Geneva, Switzerland: WHO. Available at: https:// covid19.who.int/. Accessed September 21, 2020.
2. Africa CDC, 2020. Coronavirus Disease 2019 (COVID-19) Latest Updated on the COVID-19 Crises Africa CDC. Africa CDC Dashboard. Available at: africacdc.org/covid-19/. Accessed September 20, 2021.

3. World Health Organization, 2020. Maintaining Essential Health Services: Operational Guidance for the COVID-19 Context Interim Guidance. Geneva, Switzerland: COVID-19:eSSENTIAL HEALTH SERVICES. Available at: https://www.who.int/publications//item/ WHO-2019-nCoV-essential-health-services-2020.1. Accessed September 21, 2020.

4. Elston JWT, Cartwright C, Ndumbi P, Wright J, 2017. The health impact of the 2014-15 Ebola outbreak. Public Health 143: 60-70.

5. Roberton T, Carter ED, Chou VB, Stegmuller AR, Jackson BD, Tam Y, Sawadogo-Lewis T, Walker N, 2020. Early estimates of the indirect effects of the COVID-19 pandemic on maternal and child mortality in low-income and middle-income countries: a modelling study. Lancet Glob Health 8: e901-e908.

6. Riley T, Sully E, Ahmed Z, Biddlecom A, 2020. Estimates of the potential impact of the COVID-19 pandemic on sexual and reproductive health in low-and middle-income countries. Int Perspect Sex Reprod Health 46: 73-76.

7. Shaukat N, Ali DM, Razzak J, 2020. Physical and mental health impacts of COVID-19 on healthcare workers: a scoping review. Int J Emerg Med 13: 40.

8. National Population Commission (NPC) [Nigeria], ICF, 2020. Nigeria Demographic Health Survey 2018. Rockville, MD: DHS Program ICF Rockville Rockville.

9. Nigeria Centre for Disease Control, 2020. COVID-19 Situation Report-186. An update of COVIID-19 outbreak in Nigeria NCDC 01 September 2020. Available at: ncdc.gov.ng/diseases/sitreps/. Accessed September 21, 2020.

10. Semaan A et al., 2020. Voices from the frontline: findings from a thematic analysis of a rapid online global survey of maternal and newborn health professionals facing the COVID-19 pandemic. BMJ Glob Health 5: e002967.

11. Ezenwa BN, Fajolu IB, Akinajo OR, Makwe CC, Oluwole AA, Akase IE, Afolabi BB, Ezeaka VC, 2020. Management of COVID-19: a practical guideline for maternal and newborn health care providers in sub-Saharan Africa. J Matern Neonatal Med 1-7. Available at: https://doi.org/10.1080/14767058.2020.1763948.

12. Poon LC et al., 2020. Global interim guidance on coronavirus disease 2019 (COVID-19) during pregnancy and puerperium from FIGO and allied partners: information for healthcare professionals. Int J Gynecol Obstet 149: 273-286.

13. Zafar N, Jamal Z, Mujeeb Khan M, 2020. Preparedness of the healthcare personnel against the coronavirus disease 2019 (COVID-19) outbreak: an audit cycle. Front Public Health 8: 502.

14. Elhadi $M$, Msherghi $A$, Elgzairi $M$, Alsuyihili A, Elkhafeefi $F$, Bouhuwaish A, Biala M, 2020. Assessment of the preparedness of obstetrics and gynecology healthcare systems during the COVID-19 pandemic in Libya. Int J Gynecol Obstet 150: 406-408.

15. Elhadi M et al., 2020. Assessment of healthcare workers' levels of preparedness and awareness regarding COVID-19 infection in low-resource settings. Am J Trop Med Hyg 103: 828-833.

16. UN Habitat, 2020. Lagos: Citizen Participation in a Multi-Cultural City Organization Details Organization: Lagos State Ministry of Physical Planning and Urban Development Organization Country: Nigeria Other Organization Name. Available at: https:// wuf.unhabitat.org/sites/default/files/2020-01/VC A 27.pdf. Accessed September 21, 2020.

17. Ministry of Health Lagos state Nigeria, 2020. Health Facility Monitoring And Accreditation Agency (Hefamaa) Assessment Tool - Ministry of Health Lagos. Available at: https:/health.lagosstate.gov.ng/2017/ 06/02/health-facility-monitoring-and-accreditation-agency-hefamaaassessment-tool. Accessed September 21, 2020.

18. Hosmer DW, Lemeshow S, Sturdivant RX, 2013. Applied Logistic Regression, 3rd edition. Hoboken, NJ: Wiley.

19. Kristensen TS, Borritz M, Villadsen E, Christensen KB, 2005. The copenhagen burnout inventory: a new tool for the assessment of burnout. Work Stress 19: 192-207.

20. Pallant JF, 2020. SPSS Survival Manual: A Step by Step Guide to Data Analysis Using IBM SPSS, 6th edition. Sydney, Australia: Open University Press. 
21. Menard S, 2014. Logistic Regression: from Introductory to Advanced Concepts and Applications. Los Angeles, CA: Sage.

22. Tabachnick BG, Fidell LS, 2012. Using Multivariate Statistics, 6th edition. New York, NY: Harper \& Row.

23. Louviere JJ, Hensher DA, Swait JD, Adamowicz W, 2000. Stated Choice Methods: Analysis and Application. Cambridge, UK: Cambridge University Press.

24. Suleiman A et al., 2020. Preparedness of frontline doctors in Jordan healthcare facilities to COVID-19 outbreak. Int J Environ Res Public Health 17: 3181.

25. McMahon SA, Ho LS, Brown H, Miller L, Ansumana R, Kennedy CE, 2016. Healthcare providers on the frontlines: a qualitative investigation of the social and emotional impact of delivering health services during Sierra Leone's Ebola epidemic. Health Policy Plan 31: 1232-1239.

26. Almutairi AF, Adlan AA, Balkhy HH, Abbas OA, Clark AM, 2018. "It feels like I'm the dirtiest person in the world": exploring the experiences of healthcare providers who survived MERS-CoV in Saudi Arabia. J Infect Public Health 11: 187-191.

27. Onigbogi $C$, Banerjee $S, 2019$. Prevalence of psychosocial stress and its risk factors among health-care workers in Nigeria: a systematic review and meta-analysis. Niger Med $J$ 60: 238-244.

28. Adesegun OA et al., 2020. The COVID-19 crisis in sub-Saharan Africa: knowledge, attitudes, and practices of the Nigerian public. Am J Trop Med Hyg 103: 1997-2004.

29. Witter S, Palmer N, Balabanova D, Mounier-Jack S, Martineau T, Klicpera A, Jensen C, Pugliese-Garcia M, Gilson L, 2019. Health system strengthening-Reflections on its meaning, assessment, and our state of knowledge. Int $J$ Health Plann Manage 34: e1980-e1989.

30. Wilson AN et al., 2020. Caring for the carers: ensuring the provision of quality maternity care during a global pandemic. Women Birth. Available at: https://doi.org/10.1016/j.wombi. 2020.03.011.

31. Witter S et al., 2017. How do health workers experience and cope with shocks? Learning from four fragile and conflict-affected health systems in Uganda, Sierra Leone, Zimbabwe and Cambodia. Health Policy Plan 32 (Supp/ 3): iii3.

32. Partnership for Evidence-Based Response to COVID-19, 2020. Finding the Balance: Public Health and Social Measures in Nigeria. Available at: https://preventepidemics.org/wp-content/ uploads/2020/09/09082020-nigeria.pdf. Accessed September 25, 2020.

33. Akande-Sholabi W, 2020. Nigeria's Reliance on Drug Imports in the Time of COVID-19. Medical Press. Available at: https:// medicalxpress.com/news/2020-04-nigeria-reliance-drugimports-covid-.html. Accessed September 25, 2020.

34. Olusanya BO, Roberts AA, Olufunlayo TF, Inem VA, 2010. Preference for private hospital-based maternity services in innercity Lagos, Nigeria: an observational study. Health Policy (New York) 96: 210-216.

35. Anastasi E, Ekanem E, Hill O, Adebayo Oluwakemi A, Abayomi O, Bernasconi A, 2017. Unmasking inequalities: sub-national maternal and child mortality data from two urban slums in Lagos, Nigeria tells the story. PLoS One 12: e0177190. 